International Journal of Design and Manufacturing Technology (IJDMT), ISSN 0976 - 6995(Print), ISSN 0976 - 7002(Online)

Volume 1, Issue 1, January-December (2010), pp. 14-23

(C) IAEME, http://www.iaeme.com/ijdmt.html

\title{
GENERAL FORMULATION FOR A MANUFACTURING OF DEFECTIVE SETUP WITH DIFFERENT ADJUSTMENT METHODS
}

\author{
S Y Gajjal \\ Asst. Prof., SCOE, Pune
}

\author{
Dr. A P S Gaur \\ Prof. B.U, Jhansi
}

\author{
Dr. S R Kajale \\ Director, SGGS, Nanded
}

\begin{abstract}
Consider the problem of adjusting a machine that starts production after a defective setup operation. This setup adjustment problem was first studied by Grubbs (1954, 1983). A general formulation for setup adjustment problems is presented in this paper. The formulation unifies some well-known process adjustment schemes, including Grubbs' harmonic and extended rules, adjustment methods based on stochastic approximation and recursive least squares, and a recent method on adaptive EWMA feedback controllers. The proposed formulation is Bayesian and based on a Kalman Filter. The formulation allows to show the equivalence of the setup process adjustment problem with a simple instance of what in the control engineering literature is called a Linear Quadratic Gaussian (LQG) controller. As an important byproduct, the LQG model allows to solve more complicated setup adjustment problems with readily available techniques. Extensions to cases when there are quadratic adjustment costs and when the problem is multivariate are discussed. The multivariate setup adjustment solution is illustrated with a multihead filling machine example.
\end{abstract}

\section{INTRODUCTION}

Process adjustment techniques based on the feedback principle have become an important resource in the toolkit used by Quality Engineers (see, for example, Box et al., 1994, Box and Luceño, 1997, Sachs et al., 1995). A variety of techniques for process adjustment have been proposed and studied, partly due to interest on integration of Statistical Process Control (SPC) and Engineering Process Control (EPC) methods. This paper presents a formulation that unifies several well-known process adjustment schemes and shows how several extensions can be obtained using standard methods from control engineering.

This paper is organized as follows. Section 2 summarizes the two adjustment rules proposed by Grubbs. A Bayesian formulation of the setup adjustment problem based on a Kalman Filter is presented in section 3. There it is shown that the Kalman Filter controller contains as particular cases several other adjustment techniques. Section 4 presents extensions of the basic model to more complex setup adjustment 
International Journal of Design and Manufacturing Technology (IJDMT), ISSN 0976 - 6995(Print), ISSN 0976 - 7002(Online) Volume 1, Issue 1, January-December (2010), @ IAEME

problems. These include the multivariate case, which is numerically illustrated with application to a multiple spindle machine process.

\section{GRUBBS' ADJUSTMENT RULES}

In this section an explanation of Grubbs $(1954,1983)$ process adjustment rules is provided with terminology and notation that will allow comparison with other methods discussed in later sections. Following Grubbs, assume initially that the setup offset $d$ is an unknown constant, not necessarily equal to zero. Then for the first manufactured part the mean deviation from the target of the quality characteristic is assumed equal to:

$$
\mu_{l}=d+U_{0}
$$

where $U_{0}$ is the initial setting of the machine setpoint (the controllable factor). Grubbs assumed that $U_{0}=0$, i.e., it was assumed that before start-up the machine is aimed at the target, since there is no information yet available about the offset $d$. If $d$ were perfectly known, the setting $U_{t}=-d$ for all $t$ will completely eliminate the offset; this trivial case is excluded in what follows.

The first observed deviation from target will be given by

$$
Y_{1}=\mu_{1}+v_{1}=d+U_{0}+v_{1}
$$

Where the $v_{t}$ 's are assumed identically and independently distributed as a normal with mean 0 and variance $\sigma_{v}^{2}$ and models both the part-to-part variability and the measurement error. As noted by Trietsch (1998), these two variance components always appear together in this type of models so for simplicity of notation no distinction between them will be made.

It will be assumed that adjustments affect the mean of the process. This is in sharp contrast with some EPC methods where the adjustment is supposed to modify the observed deviation, simply compensating for some inherent deviation that cannot be corrected (Del Castillo, 1998). Thus, in our case, the first set point adjustment $\nabla U_{l}=$ $U_{l}-U_{0}$ will result in a new process mean of

$$
\mu_{2}=\mu_{1}+\nabla U_{1}
$$

and the second deviation from target will be

$$
Y_{2}=\mu_{2}+v_{2}
$$

Continuing in this form, the general expressions for the mean of the process and the deviation from target are, respectively,

$$
\mu_{t}=\mu_{t-1}+\nabla U_{t-1}
$$

and

$$
Y_{t}=d+U_{t-1}+v_{t}
$$

Or, equivalently:

$$
Y_{t}=\mu_{t}+v_{t}
$$


International Journal of Design and Manufacturing Technology (IJDMT), ISSN 0976 - 6995(Print), ISSN 0976 - 7002(Online) Volume 1, Issue 1, January-December (2010), @ IAEME

Grubbs $(1954,1983)$ proposed to find the adjustment weights $\left\{K_{i}\right\}_{i=1}^{n}$ such that the linear adjustment policy $\nabla U_{t}=-K_{t} Y_{t}$ solves the following problem for any finite number of observations $n>0$ :

$$
\begin{gathered}
\min \operatorname{Var}\left(\mu_{n+1}\right) \\
\text { Subject to: } E\left[\mu_{n+1}\right]=0 .
\end{gathered}
$$

Note that the form of the control rule is fixed first and then the rule is optimized by solving (6). We also point out that $\mu_{t}$ becomes a random variable since it is a function of adjustments based on random observations.

In an elegant derivation, Grubbs show that, provided nothing is known a priori about the magnitude of the offset, the weights $K_{t}$ that solve problem (6) satisfy

So the adjustments are simply

$$
K_{t}=1 / t \quad t=1,2, \ldots
$$

$$
\nabla U_{t}=U_{t}-U_{t-1}=-\frac{Y_{t}}{t^{x}}
$$

Thus after producing the first part, the machine setpoint is adjusted by the full observed deviation. After the second part is produced, the set point is adjusted by half the observed deviation from target, and so on. The adjustment constants follow the harmonic series $\{1,1 / 2,1 / 3, \ldots\}$. For this reason, Trietsch (1998) calls this adjusting scheme the "harmonic rule".

\section{Grubbs extended rule}

A second adjustment rule was also proposed by Grubbs. In this case, the setup offset $d$ is modeled as a random variable instead of as an unknown constant. Grubbs assumed the distribution of $d$ has a known mean equal to zero and a known variance $\sigma$ ${ }_{d}^{2}$. According to him, the "variance of setup" $\left(\sigma_{\mathbb{t}}^{2}\right)$ is due to changes in the machine over many "occasions", an occasion understood as one setup operation. He then shows that the optimal weights that solve problem (6) for these new assumptions are given by

$$
K_{t}=\frac{1}{t+\frac{\sigma_{0}^{2}}{\sigma_{\mathbb{d}}^{2}}}
$$

and the adjustments are $\nabla U_{t}=-K_{t} Y_{t}$. Following Trietsch (1998) we refer to this rule as "Grubbs extended rule".

It is important to understand what type of optimality claims one can make for Grubbs' rules. These are explained next, where a more general formulation is presented.

\section{A KALMAN FILTER MODEL FOR PROCESS ADJUSTMENT}

Consider the more general case when the setup error $d$ is a random variable with known mean $\bar{d}$ and known variance $P_{0}$. Suppose the objective is to find the process adjustments $\nabla U_{1}, \nabla U_{2}, \ldots, \nabla U_{n-1}$ that minimize

$$
E\left[\sum_{t=1}^{n} \mu_{t}^{2}\right]
$$


International Journal of Design and Manufacturing Technology (IJDMT), ISSN 0976 - 6995(Print), ISSN 0976 - 7002(Online) Volume 1, Issue 1, January-December (2010), @ IAEME

In other words, it is assumed that there are quadratic off-target costs but no cost is incurred when performing the adjustments. Optimization of this type of criterion is based on the separation principle (Åström, 1970, Lewis, 1986). For the setup adjustment problem under consideration, this principle indicates that the optimal solution can be found by solving separately the problem of estimating the $\mu_{t}$ 's (process means) from the problem of finding the best adjustments $\left\{\nabla U_{t}\right\}$. If the optimal adjustment equation that is obtained through this separation is identical to what would have been obtained if the process were deterministic, the controller is said to be a certainly equivalence controller. This essentially means that the parameter estimates are used in the control equation as if they were the true parameters. In our case, if the means $\mu_{t}$ were known it is evident that the best adjustment would be simply to set $\nabla U_{t}=-\mu_{t}$ which from equations (3-5) yields a minimum variance process which is on-target on average. In what follows, it is shown that Grubbs' extended rule is a simple case of a certainty equivalence controller in which we adjust by $\nabla U_{t}=-\hat{\mu}_{\mathrm{t}}$ where the mean $\mu_{t}$ is estimated separately using a Kalman filter.

It has been shown that certainty equivalence holds for processes that obey linear difference equations (such as eq. (3)) when the criterion to optimize is a quadratic function of the state variable and the control factor $\left(\mu_{t}\right.$ and $\nabla U_{t}$ in our case). This is the celebrated Linear Quadratic (LQ) control problem. The Appendix shows the solution to a more general LQ control problem applied to setup adjustment problems. We now show how Grubbs' extended rule is the optimal solution for criterion (9). Recently, Trietsch (1998) pointed out the optimality of Grubbs' extended rule. We emphasize that this optimality property only holds if the first two moments of the setup error distribution are known.

The estimation problem is solved in a Bayesian framework using a simple Kalman filter (Meinhold and Singpurwalla, 1983). Given the model (3-5), define

$$
Y^{t} \mid \mu_{t} \sim N\left(\mu_{t}, \sigma_{\vartheta}^{2}\right)
$$

and define the prior distribution of $\mu_{t}$ :

$$
\mu_{t} \mid Y^{t-1} \sim N\left(\hat{\mu}_{t-1}+\nabla U_{t-1}, P_{t-1}\right)
$$

where $Y^{t}=\left\{Y_{1}, Y_{2}, \ldots, Y_{t}\right\}$ are all available data at time $t$. The first mean has a prior distribution $\mu_{t} \sim N\left(\bar{d}+U_{0}, P_{0}\right)$ where $\bar{d}=E[d], \operatorname{Var}[d]=P_{0}$ and $U_{0}$ is the initial setpoint of the machine. Note that if $\bar{d}$ is known, then we should set $U_{0}=-\bar{d}$ and get $\mu_{t} \sim N(0$, $\left.P_{0}\right)$.

The posterior mean of $\mu_{t}$ is $\widehat{\mu}_{\mathrm{t}}=E\left[\mu_{t} \mid Y^{t}\right]$, and the posterior variance of $\mu_{t}$ is defined as

$$
P_{t}=\operatorname{Var}\left(\mu_{t} \mid Y^{t}\right) \text {. }
$$

Given this setup, we have that

$$
\mu_{t} \mid Y^{t} \sim N\left(\hat{\mu}_{t}, P_{t}\right)
$$

with

$$
\hat{\mu}_{\mathrm{t}}=\hat{\mu}_{\mathrm{t}-1}+\nabla U_{t-1}+\frac{P_{t-1}}{P_{t-1}+\sigma_{v}^{2}}\left[Y_{t}-\left(\hat{\mu}_{\mathrm{t}-1}+\nabla U_{t-1}\right)\right]
$$

and 
International Journal of Design and Manufacturing Technology (IJDMT), ISSN 0976 - 6995(Print), ISSN 0976 - 7002(Online) Volume 1, Issue 1, January-December (2010), @ IAEME

$$
P_{t}=\frac{P_{t-1} \sigma_{v}^{2}}{\sigma_{w}^{2}+P_{t-1}}
$$

Which is a recurrence equation easily solved by iteration yielding

$$
P_{t}=\frac{\sigma_{v}^{\mathrm{x}} P_{0}}{\sigma_{\mathrm{v}}^{\mathrm{z}}+t P_{0}}
$$

Where $P_{0}$ is known. The Kalman filter estimate of the process mean given the data is

$$
E\left[\mu_{t} \mid Y^{t}\right]=\hat{\mu}_{\mathrm{t}}=\hat{\mu}_{\mathrm{t}-1}+\nabla U_{t-1}+K_{t}\left[Y_{t}-\left(\hat{\mu}_{\mathrm{t}-1}+\nabla U_{t-1}\right)\right]
$$

Where the quantities

$$
K_{t}=\frac{P_{t-1}}{P_{t-1}+\sigma_{v}^{2}}=\frac{1}{t+\frac{\sigma_{v}^{2}}{P_{0}}}
$$

are the "Kalman weights". Note that expression (10) is independent of the particular choice of the $\nabla U_{t}$ 's (a consequence of the separation principle). Under the stated assumptions of normality, $\hat{\mu}_{\mathrm{t}}$ is the minimum mean square error (MMSE) estimator of $\mu_{t}$. As shown by Duncan and Horn (1972), if the normality assumptions are relaxed, $\hat{\mu}_{\mathrm{t}}$ is the MMSE linear estimator, i.e., among all estimators that are linear combinations of the observations it has smallest MSE, but there may be better nonlinear estimators.

To minimize $E\left[\sum_{t=1}^{n} \mu_{t}^{2}\right]$ one can argue as follows. Conditioning on all available data at time $t-1$ we have that

$$
\begin{gathered}
E\left[\mu_{t}^{2} \mid Y^{t-1}\right]=\operatorname{Var}\left(\mu_{t} \mid Y^{t-1}\right)^{2}+\left[E\left(\mu_{t} \mid Y^{t-1}\right)\right]^{2} \\
=\frac{\sigma_{v}^{s} p_{0}}{\sigma_{v}^{2}+(t-1) P_{0}}+\left(\hat{\mu}_{\mathrm{t}-1}+\nabla U_{t-1}\right)^{2}
\end{gathered}
$$

which is minimized by taking $\nabla U_{t-1}=-\hat{\mu}_{\mathrm{t}-1}$. From our earlier discussion, this is a certainty equivalence controller. Applying this adjustment rule at every point in time also minimizes the sum of the squared deviations (9). This can be formally proved by showing how this problem is a particular instance of a LQ problem and utilizing the LQ solution (see Appendix).

Substituting the control rule into the process mean estimate, we get

$$
\widehat{\mu}_{\mathrm{t}}=\frac{1}{t+\frac{\sigma_{v}^{g}}{P_{0}}} Y_{t}=K_{t} Y_{t}
$$

and the adjustment rule is

$$
\nabla U_{t}=\frac{-Y_{t}}{t+\frac{\nabla_{v}^{2}}{P_{0}}}
$$

We conclude that Grubbs' extended rule minimizes the expected sum of squared deviations provided the setup error mean and variance are known. If the errors are all normally distributed, Grubbs extended rule is the optimal solution for criterion (9). If the errors are not normal, Grubbs extended rule is the best linear control law that 
International Journal of Design and Manufacturing Technology (IJDMT), ISSN 0976 - 6995(Print), ISSN 0976 - 7002(Online) Volume 1, Issue 1, January-December (2010), @ IAEME

minimizes (9) ( ̊̊ström, 1970, Lewis, 1986). These additional facts can also be proved using LQ or LQG (linear control gaussian) theory.

Besides the aforementioned equivalence with Grubbs' extended rule, the Kalman filter equation (11) together with equation (12) provide three other particular cases of interest:

1. Grubbs harmonic rule and Robbins and Monro stochastic approximation.-

If $P_{0} \rightarrow$, which implies lack of any a priori information on the offset $d$, Grubbs" "harmonic rule" is obtained, since, under these conditions the Kalman weights

$$
K_{t} \rightarrow \frac{1}{t}
$$

The mean estimates become

$$
\widehat{\mu}_{t}=\frac{1}{t} Y_{t}
$$

and

$$
\nabla U_{\mathrm{t}}=-\frac{1}{t} Y_{t}
$$

or

$$
U_{t}=U_{t-1}-\frac{1}{t} Y_{t}
$$

which is exactly Grubbs' harmonic rule.

Grubbs obtained (13) by solving a constrained optimization problem (i.e., min $\operatorname{Var}\left(Y_{t+1}\right)$, subject to $E\left[Y_{t+1}\right]=0$ ) under the assumption the setup error $d$ was an unknown constant ( a machine offset). If $d$ is an unknown constant, we have from (2) that

$$
\mu_{1}=d+U_{0}
$$

and from (4) and (5)

$$
\mu_{t}=d+U_{t-1}
$$

Thus, if $d$ were a known constant the best controller would be simply $U_{t}=-d$ and the certainty equivalence controller if $d$ is unknown is $U_{t}=$ - where is an estimate of the setup offset $d$ at time $t$. From (13) we have that

$$
\begin{array}{r}
U_{t}=-i=U_{t-1}-Y_{t} / t=-\hat{\mathrm{d}}_{\mathrm{t}-1} \cdot Y_{t} / t, \text { or } \\
\hat{d}_{\mathrm{t}}=\hat{d}_{\mathrm{t}-1}+K_{t} Y_{t}
\end{array}
$$

With $K_{t}=1 / t$.

Interestingly, equation (14) allows to see that Grubbs' harmonic rule is a special case of Robbins and Monro's (1951) stochastic approximation algorithm for the sequential estimation of the offset $d$. Robbins and Monro's procedure was obtained by investigating the conditions under which the sequence $K_{t}$ makes $\lim _{n \rightarrow \infty} E[d-d]^{2}=0$ (i.e., the mean square convergence 
International Journal of Design and Manufacturing Technology (IJDMT), ISSN 0976 - 6995(Print), ISSN 0976 - 7002(Online) Volume 1, Issue 1, January-December (2010), @ IAEME

of the estimates dto ). Later Blum (1954) established the stronger result of convergence with probability one for the Robbins-Monro scheme. Since then, a wealth of asymptotic result have been reported with respect to Robbins and Monro's procedure and its modification. The setup adjustment application requires a study of the finite sample properties of these methods for the relatively simple problem under consideration, that of sequentially estimating an unknown scalar d. Such a study is reported in Del Castillo, Pan and Colosimo (2002).

2. Recursive least squares.- If $c$ is set equal to one and $K_{t}=1 /\left(t+\sigma_{2}^{2} P_{0}\right)$ is substituted in (14), the so-called recursive least squares (Young, 1984, Ljung and Söderström, 1987) estimate of $d$ is obtained:

$$
\hat{d}_{t}=\hat{d}_{t-1}+\frac{1}{\frac{1}{P_{0}}+t} Y_{t} .
$$

In this case we are again assuming the first case studied by Grubbs, that of an unknown constant setup error $d$.

\section{EXTENSIONS TO MORE COMPLEX SETUP ADJUSTMENT PROBLEMS}

It was mentioned in the previous section that the Kalman filter model that yields Grubbs' extended rule (equation 11-12) is optimal for the criterion $E\left[\sum_{t=1}^{n} \mu_{t}^{2}\right]$ and this constitutes a simple instance of a linear quadratic control problem. The more general LQ formulation of the appendix allows to derive optimal adjustment rules for more complex setup adjustment problems. In particular, consider the following cases:

1. Errors in the adjustments. In this case, the process mean obey

$$
\mu_{t}=\mu_{t-1}+U_{t-1}+\omega_{t}, \quad \omega_{t} \cdot N\left(0, \quad \sigma_{t}^{2}\right.
$$

where $U_{t-1}+\omega_{t}$. is the actual adjustment made at time $t$, which equal the desired adjustment $\left(U_{t-1}\right)$ plus some adjustment error. Errors in the adjustments were considered by Trietsch (1998).

2. Quadratic adjustment costs. If the cost of an adjustment is quadratic, the optimization criterion is changed to

$$
\min E \quad\left[\sum_{t-1}^{n-1}\left[Q_{\Re}+R\left(U_{t}\right)^{2}\right]+Q_{\Re}\right]
$$

Where the off-target cost and $R$ is is the adjustment cost.

3. Multiple controllable factors (setpoints), multiple quality characteristics.

Suppose there are $p$ controllable factors (e.g., setpoints) $\boldsymbol{U}_{t}$ that have an immediate effect on $p$ quality characteristics (deviations from target) $\boldsymbol{Y}_{\boldsymbol{t}}$. Assume there are no adjustment costs $(R=0)$ or errors in the adjustments ( $\sigma_{\omega}^{2}=C$. The optimization criterion is then

$$
\min E\left[\sum_{t=1}^{n} \mu_{t}^{\sigma}\right]
$$

where $\boldsymbol{\mu}_{t}$ is a $p \quad 1$ vector of process mean. The LQ formulation in the Appendix yields in this case a multivariate adjustment rule that is the exact multivariate analogy of Grubbs' extended rule:

$$
\begin{aligned}
& \boldsymbol{\mu}_{t}=\boldsymbol{\mu}_{t-1}+\boldsymbol{U}_{t-1} \\
& \boldsymbol{Y}_{t}=\boldsymbol{\mu}_{t}+\boldsymbol{v}_{t}, \quad \boldsymbol{v}_{t} \sim N\left(0, \sum_{\mathrm{v}}\right) \\
& \widehat{\boldsymbol{\mu}}_{\mathrm{t}}\left(t I+\sum_{\mathrm{v}} \quad P\right)^{-1} \boldsymbol{Y}_{t}=K_{t} \boldsymbol{Y}_{t}
\end{aligned}
$$

and the $p$ adjustments are given by 
International Journal of Design and Manufacturing Technology (IJDMT), ISSN 0976 - 6995(Print), ISSN 0976 - 7002(Online) Volume 1, Issue 1, January-December (2010), @ IAEME

$$
\boldsymbol{U}_{t}=\left(\begin{array}{ll}
t I+\sum_{v} & P
\end{array}\right)^{-1} \boldsymbol{Y}_{t}
$$

Where the initial setup error $\boldsymbol{d}$ follows a multivariate distribution with mean and variance matrix $\sum_{d}=P_{0}$.

Example.- (A multiple stream process.) Consider the application of the multivariate adjustment scheme to a bottle filling process in which measurements of the fill weight or volume generated by each of three spindles are measured. The spindles may be offtarget after setting up the machine, and each may be off by different amounts and directions. Suppose it is known from historical records that the following variancecovariance matrix of the deviations target is available

$$
\sum_{v}+\left[\begin{array}{ccc}
5 & 3.5 & 2.7 \\
3.5 & 3 & 2.5 \\
2.7 & 2.5 & -2.8
\end{array}\right]
$$

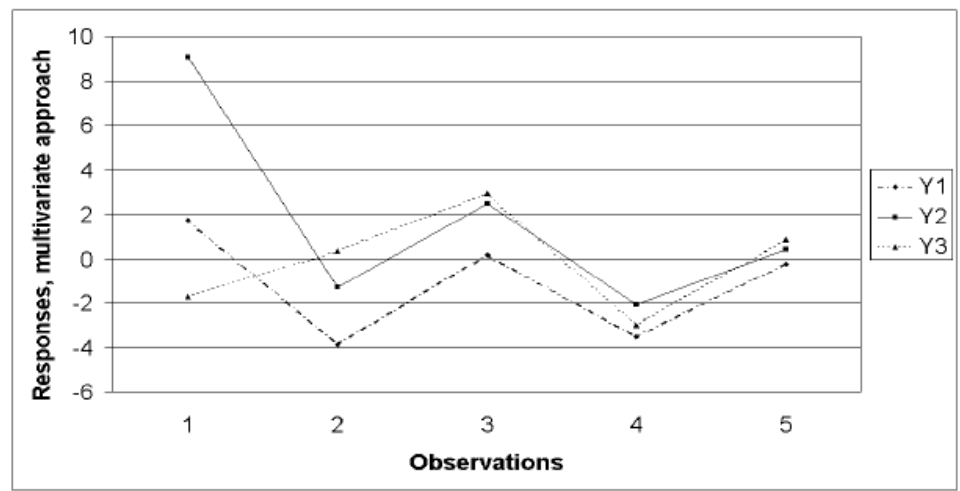

Figure 1 : Simulated multiple spindle process adjusted with multivariate Kalman filter

Suppose that the initial machine settings are $\boldsymbol{U}_{\boldsymbol{0}}=\mathbf{0}$, and assume $\boldsymbol{d} \sim N\left(\overline{\boldsymbol{d}}, P_{0}\right)$ $=N\left((0,10,0)^{\prime}, 5 I\right)$ (note how the initial setting $\left.\boldsymbol{U}_{\boldsymbol{0}} \neq \overline{\boldsymbol{d}}\right)$. Then we have that the first Kalman matrix is given by:

$$
K_{l}=\left[\sum_{v} P_{0}^{-1}+I\right]^{-1}=\left[\begin{array}{ccc}
0.6172 & -0.2259 & -0.1412 \\
-0.2259 & 0.7772 & -0.1709 \\
-0.1412 & -0.1709 & 0.7447
\end{array}\right]
$$

Using simulated values according to the assumed data, in this problem, suppose the first observed vector of deviations from target is $Y_{1}^{e}=(1.7587,9.0797,-1.7250)$, where the error terms $v_{t}$ were simulated from a multivariate normal distribution with mean zero and variance matrix $\sum_{v}$. With this, we have that,

$$
\nabla \boldsymbol{U}_{l}=K_{l} \boldsymbol{Y}_{1}, \quad \text { or } \quad \boldsymbol{U}_{\boldsymbol{l}}=\boldsymbol{U}_{\boldsymbol{0}}-K_{l} \boldsymbol{Y}_{1}=(0.7218,-6.9546,3.0850)^{g}
$$

Figure 1 illustrates the deviations from target of such simulated process for the first 5 observations. 
International Journal of Design and Manufacturing Technology (IJDMT), ISSN 0976 - 6995(Print), ISSN 0976 - 7002(Online) Volume 1, Issue 1, January-December (2010), @ IAEME

It is of interest to consider what would have happened if three univariate Kalman Filters were used for each spindle separately. Figure 2 illustrates a simulation of the first 5 deviations from target of this alternative approach, using the same random numbers used in Figure 1. As it can be seen, the convergence to target is faster if the multivariate information is used. As time passes, the differences between the two approaches are minimal, that is why the figures show only the first 5 deviations where differences are more pronounced.

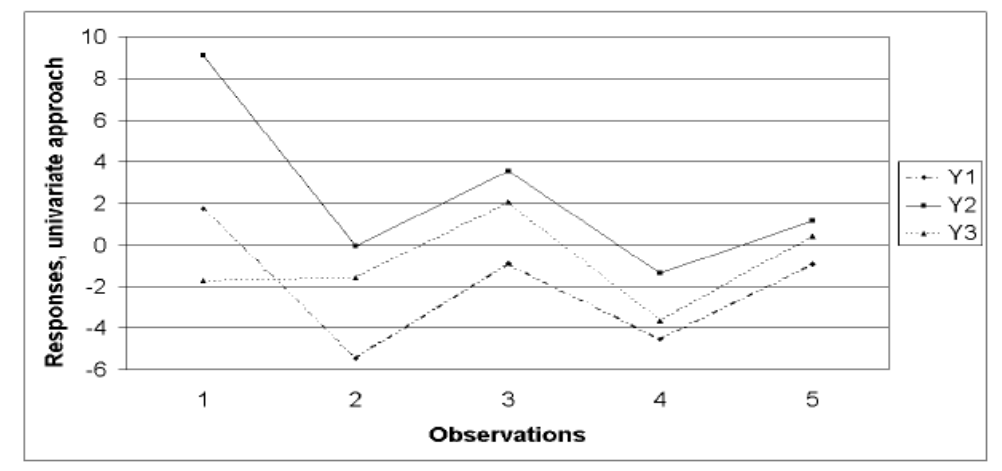

Figure 2 : Simulated multiple spindle process adjusted with three independent univariate Kalman filter scheme

\section{CONCLUSIONS}

A unifying point of view of some process adjustment procedures for setting up a machine was presented based on a Kalman Filter approach. A connection between Grubbs harmonic rule and stochastic approximation was made

It was shown how the Kalman filter adjustment scheme can be extended using LQG control theory to other more complex setup adjustment problems, such as the case there are adjustment costs, the case of errors in the adjustments, and the case the process is multivariate. The later case was illustrated showing by example the advantages of using a multivariate approach to independent Kalman filter controllers, one per quality characteristic.

\section{REFERENCES}

1. Karl J. Astrom., (1970). Introduction to Stochastic Control Theory. San Diego, CA: Academic Press.

2. Blum, J.R. (1954). "Approximation Methods Which Converge With Probability One," Annals of Mathematical Statistics, 25, pp. 382-386.

3. Box G.E.P., G.M. Jenkins, and Reinsel, G. (1994). Time Series Analysis, Forecasting, and Control 3rd. ed., Englewood Cliffs: Prentice Hall.

4. Box, G.E.P., Luceno, A. (1997). Statistical Control by Monitoring and Feedback Adjustment. John Wiley \& Sons, New York, NY.

5. Del Castillo, E. (1998). "A Note on two Process Adjustment Models". Quality and Reliability Engineering International, 14, pp. 23-28. 
International Journal of Design and Manufacturing Technology (IJDMT), ISSN 0976 - 6995(Print), ISSN 0976 - 7002(Online) Volume 1, Issue 1, January-December (2010), @ IAEME

6. Del Castillo, E. (2001). "Some properties of EWMA feedback quality adjustment schemes for drifting disturbances", Journal of Quality Technology, 33(2), 153166.

7. Del Castillo, E., Pan, R., and Colosimo, B.M. (2002). "Small sample comparison of some process adjustment methods", Technical Report, Dept. of Industrial Engineering, Penn State University (submitted for publication).

8. Duncan, D.B., and Horn, S.D., (1972). "Linear Dynamic Recursive Estimation from the Viewpoint of Regression Analysis," Journal of the American Statistical Association, 67, 340, pp. 815-821.

9. Grubbs, F.E., (1954, 1983). "An Optimum Procedure for Setting Machines or Adjusting Processes," Industrial Quality Control, July, reprinted in Journal of Quality Technology, 1983, 15, 4, pp. 186-189.

10. Guo, R.S., Chen, A., and Chen, J.J., (2000). "An Enhanced EWMA Controller for Processes Subject to Random Disturbances", in Moyne, J., Del Castillo E., and Hurwitz, A., eds., (2000), Run to Run Process Control in Semiconductor Manufacturing, CRC Press, Boca Raton, FL.

11. Lewis, F.L. (1986). Optimal Estimation, with an Introduction to Stochastic Control Theory. New York, NY: John Wiley \& Sons.

12. Ljung, L. and T. Söderström (1987). Theory and Practice of Recursive Identification, Cambridge, mass., The MIT Press.

13. Meinhold, R.J, and Singpurwalla N.D. (1983)., "Understanding the Kalman Filter," The American Statistician, 37, 2, 123-127.

14. Moyne, J., Del Castillo, E., and Hurwitz, A., eds., (2000), Run to Run Process Control in Semiconductor Manufacturing, CRC Press, Boca Raton, FL.

15. Patel, N.S., and Jenkins, S.T. (1999). "Adaptive Optimization of Run-to-Run Controllers: The EWMA Example" Unpublished Technical document, Texas Instruments Inc., Dallas TX.

16. Robbins, H., and Monro, S. (1951). "A Stochastic Approximation Method". Annals of Mathematical Statistics, 22, pp. 400-407.

17. Sachs, E., Hu, A, Ingolfsson, A. (1995). "Run by Run Process Control: Combining SPC and Feedback Control," IEEE Transactions on Semiconductor Manufacturing, 8,1, 26=AD 43.

18. Trietsch, D., (1998). "The Harmonic Rule for Process Adjustment with Quadratic Loss", Journal of Quality Technology, 30, 1, pp. 75-84.

19. Young, P., (1984). Recursive Estimation and Time-Series Analysis. New York: Springer-Verlag. 